Recherches en didactique des langues et des cultures

Les cahiers de l'Acedle

15-2 | 2018

Quelles médiations en didactique des langues et des cultures?

\title{
Tardieu, C. \& Eells, E. (dir., 2018), Livre Blanc de la formation en études anglophones. Commission Formations de la Société des Anglicistes de l'Enseignement Supérieur, Paris.
}

Virginie Privas-Bréauté

\section{(2) OpenEdition}

\section{Journals}

Édition électronique

URL : http://journals.openedition.org/rdlc/4187

DOI : $10.4000 /$ rdlc. 4187

ISSN : 1958-5772

Éditeur

ACEDLE

Référence électronique

Virginie Privas-Bréauté, «Tardieu, C. \& Eells, E. (dir., 2018), Livre Blanc de la formation en études anglophones. Commission Formations de la Société des Anglicistes de l'Enseignement Supérieur, Paris. », Recherches en didactique des langues et des cultures [En ligne], 15-2 | 2018, mis en ligne le 02 juin 2018, consulté le 25 septembre 2020. URL : http://journals.openedition.org/rdlc/4187 ; DOI : https://doi.org/10.4000/rdlc.4187

Ce document a été généré automatiquement le 25 septembre 2020.

Recherches en didactique des langues et des cultures is licensed under a Creative Commons AttributionNonCommercial-NoDerivatives 4.0 International License 


\title{
Tardieu, C. \& Eells, E. (dir., 2018), Livre Blanc de la formation en études anglophones. Commission Formations de la Société des Anglicistes de l'Enseignement Supérieur, Paris.
}

\author{
Virginie Privas-Bréauté
}

1 Ce premier tome du livre blanc de la formation en études anglophones a été compilé par les 13 membres de la Commission Formations de la Société des Anglicistes de l'Enseignement Supérieur (SAES) entre 2013 et 2017 puis publié en mars 2018. Il a été coordonné par deux vice-présidentes de cette société savante, Claire Tardieu (également présidente de la Commission Formations), pour la formation, et Emily Eells, pour les concours. ${ }^{1}$

2 Ce livre blanc est composé de quatre chapitres indépendants, coordonnés par une équipe d'anglicistes et intitulés comme suit :

- Le dossier MEEF (Métiers de l'Enseignement, de l'Education et de la Formation);

- Le LANSAD (LANgues pour Spécialistes d'Autres Disciplines);

- La certification CLES (Certificat de Compétences en Langues de l'Enseignement Supérieur) ;

- L'évolution du métier d'angliciste dans l'enseignement supérieur.

3 Le livre blanc s'ouvre sur un premier chapitre de trente pages consacré à la formation MEEF. L'équipe en charge de ce dossier, constituée de Stéphanie Bernard (RouenESPE), Isabelle Schmitt (Bourgogne), Maria Tang (Rennes 2), Claire Tardieu (Sorbonne Nouvelle - Paris 3) et Anne-Marie Voise (Paris-Est Créteil), rappelle tout d'abord les origines du CAPES (Certificat d'Aptitude au Professorat de l'Enseignement au Second degré) et met l'accent sur l'absence de préparation pédagogique dans les concours qui permettaient d'accéder au grade de professeur (notamment le Certificat 
d'Aptitude à l'Enseignement en Collège, CAEC, 1941-1952). L'introduction de quelques épreuves pratiques avec l'arrivée de Gustave Monod en 1950 puis les diverses réformes successives ont permis au concours, qui prit le nom de CAPES en 1952, de devenir progressivement professionnalisant. Puis, dès 1991, apparurent sous le ministère de Lionel Jospin les IUFM (Instituts Universitaires de Formation des Maitres) qui confirmèrent ce désir de professionnaliser les fonctionnaires stagiaires mais présentèrent quelques limites : un difficile rapprochement des écoles normales et des universités, un fonctionnement tricéphale (rectorat, IUFM, université), une absence de centre de recherche et de laboratoire, une non-délivrance de diplôme. Les auteurs font ensuite un retour sur l'évolution positive de la formation des enseignants du second degré pour parvenir à la mastérisation introduite en 2010, la création des ESPE (Ecole Supérieure du Professorat et de l'Education, en remplacement des IUFM) en 2013, puis la rénovation du CAPES en 2014. Le sujet de la mastérisation leur tenant particulièrement à cœur, les membres de cette commission se sont penchés sur les maquettes des Masters MEEF mention second degré depuis 2014 au sein des universités françaises en menant deux enquêtes (en 2014 puis en 2015) auprès des adhérents de la SAES concernés par le sujet. Les deux synthèses claires, précises et chiffrées de ces questionnaires (dont l'un est en annexe du dossier) laissent apparaitre une grande hétérogénéité aussi bien dans la mise en place que dans l'administration des formations proposées tendant vers une progressive harmonisation des pratiques en 2015 . Ce chapitre se clôt sur les préconisations de la commission afin de pérenniser certains éléments des formations actuelles (les stages, la formation en didactique, la recherche, les liens entre les ESPE, les universités et les rectorats) et trois pistes d'amélioration de la formation (introduction d'une mention "pratiques et ingénierie de la formation ", pré-professionnalisation en licence, allègement de l'année de Master 2). Dans un contexte de réflexion autour de la refonte possible du CAPES, ces pistes pourront être exploitées.

4 Cédric Brudermann (Sorbonne Université), Marie-Annick Mattioli (Paris Descartes IUT), Anne-Marie Roussel (Ecole Normale Supérieure de Paris-Saclay), et Cédric Sarré (Sorbonne Université ESPE) sont les auteurs du chapitre suivant dédié au secteur LANSAD et couvrant 24 pages. Ce dossier vise à mettre au jour les enjeux de l'enseignement/apprentissage des langues étrangères dans l'enseignement supérieur auprès d'étudiants spécialistes d'autres disciplines que les langues. Aussi, une enquête nationale a été lancée en 2015 auprès de 80 responsables de structures LANSAD. Les auteurs présentent deux documents dans ce dossier : le document $n^{\circ} 1$ est une synthèse de l'enquête tandis que le document $n^{\circ} 2$ détaille les résultats publiés dans la revue Recherche et Pratiques Pédagogiques en Langues de Spécialité en 2016 (vol. 35, numéro spécial 1).

5 La synthèse qualitative et quantitative de l'étude sur le secteur LANSAD montre la primauté de l'anglais dans les offres en langues dans l'enseignement supérieur. Les auteurs indiquent les similitudes et différences entre les divers départements et le traitement du LANSAD dans les universités et précisent que les données sont variables en fonction des environnements, des équipes et des étudiants concernés. Au vu des résultats, ils déplorent l'insuffisance de lien entre la recherche et l'enseignement dans le secteur LANSAD et concluent ce premier document en soulignant l'insuffisance des moyens en matière d'encadrement par du personnel titulaire et spécialiste du secteur. Ils encouragent le recrutement de spécialistes en didactique ou en anglais de spécialité, 
soumettent l'idée d'ouvrir un concours spécifique pour les personnels du second degré afin de former les futurs enseignants d'anglais au LANSAD et envisagent le recrutement d'enseignants-chercheurs afin d'enrichir l'offre de formation au niveau Master.

6 Le second document, dont la reproduction intégrale dans ce livre blanc a été accordée par la revue Recherche et Pratiques Pédagogiques en Langues de Spécialité, reprend précisément la démarche des auteurs de l'enquête afin de faire ressortir, de manière quantifiée, tous les enjeux du secteur LANSAD en termes d'identité des structures, de politique des langues dans le secteur, de besoins et perspectives en matière de formation, d'encadrement et de recrutement. Le lecteur peut trouver une cartographie des établissements ayant participé à l'enquête en annexe du dossier.

7 Le chapitre suivant, de 9 pages, concerne la certification CLES et a été intégralement rédigé par Cédric Sarré, Directeur-adjoint de la coordination nationale CLES. Dans son introduction, Sarré explique que la naissance du CLES en 2000 est étroitement liée à la nécessité d'évaluer toutes les compétences en langues des étudiants du LANSAD au regard des faiblesses d'autres certifications. En 2007, un arrêté (paru au BOEN du 25 avril) signe la pérennisation du CLES mais Sarré déplore le manque de visibilité de cette certification plus de deux décennies plus tard. Il articule sa présentation autour de quatre axes :

1. D'où vient-on? Dans cette partie, il remonte à la genèse du CLES.

2. Où en est-on? Il explicite les raisons de la naissance de la coordination nationale de la certification (en avril 2013) en donne le fonctionnement et les résultats pour asseoir sa légitimité (une direction nationale, un comité scientifique, un réseau et des pôles CLES, des sessions par langue et par niveau annuellement, des taux de réussite par niveau de CLES et par compétence).

3. Où va-t-on? Sarré présente la création d'une feuille de route élaborée par la Coordination nationale du CLES faisant suite à l'évaluation de l'AERES en 2013 et aux priorités de la Direction Générale de l'Enseignement Supérieur et de l'Insertion Professionnelle (DGESIP). Se dégagent 5 axes ayant pour vocation une meilleure visibilité et reconnaissance du CLES. Il note que le Ministère de l'enseignement supérieur et de la recherche soutient la démarche de la coordination nationale et le CLES dans la mesure où il représente un levier pour faire évoluer la formation en langue mais souligne les attentes fortes du Ministère en matière de taux de réussite, par exemple.

4. Pourquoi y va-t-on? Il donne ici les raisons de la persévérance des coordinateurs en ce qui concerne la valorisation de la certification.

8 Pour conclure son chapitre, l'auteur insiste sur les avantages apportés par le CLES autant pour les étudiants que pour les enseignants. Il considère le CLES comme une force qui encourage les enseignants à se poser des questions sur l'enseignement/ apprentissage des langues et à modifier leurs pratiques pédagogiques à l'université. Enfin, Sarré ne perd point de vue son objectif de promotion du CLES et livre au lecteur intéressé les coordonnées numériques de la coordination nationale en vue d'une éventuelle accréditation au CLES.

Karin Fischer (Orléans) et Linda Terrier (Toulouse Jean Jaurès), avec la contribution de Jean Albrespit (Bordeaux-Montaigne), ont collaboré à l'écriture du dernier chapitre portant sur l'évolution du métier d'angliciste dans l'enseignement supérieur. Après une présentation des 13 membres de la Commission Formations de la SAES pour en souligner la disparité en termes de sexe, âge, statut, domaine de recherche, les auteures constatent que, au-delà de leurs différences, tous sont d'accord pour affirmer que leur 
métier a évolué et que cette évolution a été influencée par l'évolution du public étudiant à former. Aussi proposent-elles d'effectuer un état des lieux des représentations sur l'évolution du métier afin de mieux en cerner les contours en 2017, puis tous les cinq ans. Elles ont ainsi diffusé un questionnaire de 9 pages (en annexe) sur les représentations des acteurs du terrain aux enseignants - membres de la SAES.

La première partie de leur étude traite du questionnaire lui-même : ses objectifs, son format, sa diffusion, son taux de participation très satisfaisant pour les auteures (401 répondants pour 380 questionnaires complétés). La deuxième partie est organisée autour des profils des répondants pour en souligner la diversité. La troisième partie fait état des résultats quantitatifs aux 51 questions fermées portant sur quatre thèmes : la recherche, l'organisation du travail, la politique d'établissement vis-à-vis des étudiants anglophones et l'enseignement. Elles classent les résultats du consensus le plus large (+ de $70 \%$ des répondants sont d'accord) aux avis les plus partagés. La quatrième partie présente quant à elle les résultats aux deux questions ouvertes pour lesquelles respectivement 354 et 356 sur 380 répondants ont répondu. A la première question ( $Q$ Quelle est selon vous la caractéristique la plus saillante de l'évolution actuelle du métier?»), 329 personnes (soit $93 \%$ ) ont porté un jugement négatif en dénonçant le manque de temps pour la recherche et le poids du travail administratif. Fischer et Terrier livrent ensuite leur méthodologie d'analyse des réponses (Allard-Poési \& Maréchal 2007) et insèrent plusieurs tableaux dans lesquels sont classées les réponses selon leurs catégories et leurs occurrences. 93,6\% des sondés ont répondu à la seconde question (« Quelle amélioration souhaiteriez-vous voir apporter en priorité ?») et ont fait apparaitre la classification par ordre d'importance suivante: rémunération, carrière, recrutement, formation, recherche, gestion administrative, conditions de travail, autres éléments divers. Les deux auteures ferment leur présentation sur le constat de la dégradation du métier d'enseignant d'anglais et proposent des préconisations. Elles donnent rendez-vous aux membres de la SAES dans cinq ans pour un nouveau questionnaire.

11 Nous avons particulièrement apprécié l'effort des auteurs à livrer les résultats des enquêtes et à en interpréter les données de manière objective. Ils n'émettent point de jugement personnel et/ou subjectif sur les données recueillies au cours des diverses enquêtes et laissent leurs lecteurs apprécier - ou non - le caractère non-orienté des questions des enquêtes et des résultats d'analyse. En outre, chaque chapitre étant accompagné de quelques notices bibliographiques, parfois partielles, nous pouvons nous demander dans quelle mesure les contributeurs de ce livre blanc ont souhaité conférer un caractère scientifique à leurs présentations.

\section{BIBLIOGRAPHIE}

Allard-Poési, F. \& Maréchal, C. (2007). « Construction de l'objet de la recherche ». In Thietart R.A. (dir.). Méthodes de recherche en management. Paris : Dunod. pp. 34-56. 


\section{ANNEXES}

\section{Liste des acronymes}

AERES : Agence d'Evaluation de la Recherche et de l'Enseignement Supérieur

BOEN : Bulletin Officiel de l'Education Nationale

CAEC : Certificat d'Aptitude à l'Enseignement en Collège

CAPES : Certificat d'Aptitude au Professorat de l'Enseignement au Second degré

CLES : Certificat de Compétences en Langues de l'Enseignement Supérieur

DGESIP : Direction Générale de l'Enseignement Supérieur et de l'Insertion

Professionnelle

ESPE : Ecole Supérieure du Professorat et de l'Education

IUFM : Institut Universitaire de Formation des Maîtres

IUT : Institut Universitaire Technologique

LANSAD : Langues pour Spécialistes d'Autres Disciplines

MEEF : Métiers de l'Enseignement, de l'Education et de la Formation

SAES : Société des Anglicistes de l'Enseignement Supérieur

\section{NOTES}

1. Il est disponible ici : http://saesfrance.org/mars-2018-publication-du-livre-blanc-de-laformation-en-etudes-anglophones/

\section{INDEX}

Thèmes : Comptes rendus

\section{AUTEUR}

\section{VIRGINIE PRIVAS-BRÉAUTÉ}

Maitre de conférences en didactique de l'anglais dans le département « Arts, Lettres et Langues » de l'Université de Lorraine, site de Nancy, et rattachée au laboratoire de recherche ATILF (UMR 7118, UL, CNRS). Ses recherches scientifiques portant sur le théâtre contemporain anglophone l'ont amenée à explorer la valeur didactique du jeu théâtral et du jeu sérieux lors de l'apprentissage/enseignement de l'anglais. Son cadrage théorique est le paradigme de l'enaction 
(neurosciences) inscrit dans la théorie des savoirs incorporés.

virginie.privas-breaute[at]univ-lorraine.fr 